\title{
The Effects of Hyperstriatal Lesions on One-Trial Passive-Avoidance Learning in the Chick
}

\author{
D. C. Davies, D. A. Taylor, and M. H. Johnson' \\ Department of Anatomy and Cell Biology, St. Mary's Hospital Medical School, London, W2 1PG, and 'M.R.C. Cognitive \\ Development Unit, London, WC1H OAH, United Kingdom
}

\begin{abstract}
The roles of different forebrain structures in memory formation in the chick were investigated using restricted radiofrequency lesions. Young chicks will spontaneously peck at a small bright bead. If the bead has been coated with a distasteful substance, the chicks learn not to peck a similar bead on subsequent presentation. Thus, in a single trial chicks learn not to peck at an aversive stimulus. Bilateral lesions of the intermediate part of the medial hyperstriatum ventrale (IMHV) prevented the acquisition of this 1-trial passiveavoidance learning task. However, neither bilateral lesions of the lateral cerebral area (LCA) nor sham operation affected learning. IMHV has previously been shown to be critically involved in the learning process of imprinting in the chick. The present experiment demonstrates that the role of IMHV in learning is not restricted to imprinting. The function of IMHV in imprinting has been suggested to be concerned with the recognition of objects. In the present study shamoperated and LCA-lesioned chicks recognized the visual characteristics of the training bead and did not avoid a bead of a different color in the test. We propose that IMHV-lesioned chicks fail to learn the task because they are unable to recognize the visual characteristics of the training bead and consequently cannot make the association between the bead and the unpleasant taste.
\end{abstract}

The domestic chick is widely used for studies of the neurobiological basis of learning and memory. In particular, filial imprinting and 1-trial passive-avoidance learning (PAL) have been investigated extensively. Imprinting is the process by which chicks learn to recognize and form a preference for the first visually conspicuous object to which they are exposed (Bateson, 1966). In 1-trial PAL, chicks learn not to peck at a bead coated with a distasteful substance (Cherkin, 1969).

Considerable effort has been directed at finding a region of the chick brain that is crucial for information storage. Autoradiography studies have demonstrated an increased incorporation of ${ }^{14} \mathrm{C}$-uracil (Horn et al., 1979) and ${ }^{14} \mathrm{C}$-2-deoxyglucose (Kohsaka et al., 1979) into a restricted part of the chick telencephalon following imprinting. This part of the chick forebrain has been defined by Horn and coworkers as the intermediate

\footnotetext{
Received Oct. 26, 1987; revised Apr. 19, 1988; accepted Apr. 25, 1988

We wish to thank B. W. Crook for technical assistance and G. Horn for constructive criticism of the manuscript.

Correspondence should be addressed to Dr. D. C. Davies, Department of Anatomy and Cell Biology, St. Mary's Hospital Medical School, Norfolk Place, Paddington, London, W2 1PG, UK.

Copyright (C) 1988 Society for Neuroscience $0270-6474 / 88 / 124662-05 \$ 02.00 / 0$
}

part of the medial hyperstriatum ventrale (IMHV: Horn, 1981, 1985). Electrophysiological changes (Payne and Horn, 1982, 1984 ) and changes in the structure of synapses have subsequently been found in IMHV following imprinting (Bradley et al., 1981; Horn et al., 1985). Furthermore, the critical importance of this region in imprinting has been investigated by lesions to IMHV. Bilateral lesions of this region prevented both the acquisition (McCabe et al., 1981) and retention (McCabe et al., 1982) of an imprinted preference. However, lesions of other forebrain regions, the dorsal part of the lateral cerebral area (LCA) and parts of the visual Wulst, did not affect imprinting (McCabe et al., 1982; Horn et al., 1983; Johnson and Horn, 1987).

Kossut and Rose (1984) have shown that the incorporation of ${ }^{14} \mathrm{C}$-2-deoxyglucose is increased in part of the medial hyperstriatum ventrale (MHV), as well as in the paleostriatum augmentatum and lobus parolfactorius, following 1-trial PAL. In addition, an enhanced incorporation of ${ }^{14} \mathrm{C}$-fucose, a radiolabeled glycoprotein precursor, has been reported in the forebrain roof (which includes the MHV) after 1-trial PAL (Sukumar et al., 1980). This enhanced incorporation was subsequently found to be associated with synaptic membranes (Burgoyne and Rose, 1980). Morphological studies (Stewart et al., 1984) have also revealed synaptic changes in part of the MHV following 1-trial PAL. Furthermore, administration of protein synthesis inhibitors into the MHV induces amnesia of a 1-trial PAL task (Patterson et al., 1986; Rose, 1987) and Mason and Rose (1987) have demonstrated electrophysiological correlates of PAL within this brain region. Thus, 3 forebrain regions have been implicated in 1-trial PAL, with most evidence pointing to the involvement of part of the MHV.

If a restricted part of the MHV is indeed necessary for 1-trial PAL, damage to this area should prevent the acquisition of the task. In addition, if the region of the MHV implicated in 1-trial PAL corresponds to the more restricted forebrain region crucial to imprinting, then bilateral lesions to IMHV should impair the former. The current study was designed to address these issues.

\section{Materials and Methods}

Twelve batches of fertile "Ross 1" eggs (Ross Poultry G.B., Ltd., Woodhall Spa. Lincs.) $5-7 \mathrm{~d}$ old were set in a paddle incubator at $37.0-38.0^{\circ} \mathrm{C}$. After $18 \mathrm{~d}$ incubation, the eggs were transferred to a hatching incubator set at $38.5-40.5^{\circ} \mathrm{C}$. The chicks hatched and were allowed to dry out in the dark. They were then transferred to individual compartments in a holding incubator $\left(33.0-35.0^{\circ} \mathrm{C}\right)$ that was housed in a quiet room and illuminated by diffuse overhead light.

Surgical procedure. Chicks, $12-18 \mathrm{hr}$ old, were anesthetized by i.p. injection of $0.12 \mathrm{ml}$ Equithesin (Davies and Horn, 1983). They wcre then assigned to 1 of 3 groups according to the treatment they were to 
receive: sham-operation, IMHV-lesions, or lesions to the LCA. Chicks that were to receive lesions were mounted in a stereotaxic instrument. The ear bars were set $8.50 \mathrm{~mm}$ above and posterior to a horizontal bar placed at the angle between the upper and lower beak. The scalp, cranium, and dura mater were incised, and a stainless steel electrode was introduced into the brain. The shaft of the electrode was insulated with varnish (Epoxylite). The lesions were made by passing a radio-frequency current through 3 sites in each hemisphere. For IMHV, the stereotaxic coordinates werc $4.00,2.75$, and $1.50 \mathrm{~mm}$ anterior to the paricto-occipital (PO) suture, respectively. All sites were $0.80 \mathrm{~mm}$ lateral to the midline and $2.50 \mathrm{~mm}$ ventral to the surface of the brain. For LCA, 3 penetrations were made in the lateral side of each hemisphere at the same anterior/posterior coordinates used for IMHV. However, the electrode tip was inserted $5.50 \mathrm{~mm}$ lateral to the midline and $1.25 \mathrm{~mm}$ below the surface of the brain (McCabe et al., 1982). After the electrode was withdrawn the skull flap was closed and the scalp sutured. The scalp of sham-operated chicks was incised and sutured in a similar manner to that of the lesioned chicks. After operation each chick was ascribed a code number, and all subsequent procedures were performed "blind," the observer not knowing the experimental history of the chicks.

Training and testing procedure. Fifteen to $20 \mathrm{hr}$ after surgery, the chicks were trained in a 1-trial PAL task similar to that described by Cherkin (1969). Each chick was placed in a cylindrical arena $30 \mathrm{~cm}$ high and $14.5 \mathrm{~cm}$ in diameter. A red bead $(6 \mathrm{~mm}$ in diameter) mounted on the end of a thin stick was dipped in the bitter-tasting substance methyl anthranilate (MeA). The bead was introduced to the chick through a hole in the arena wall $15.5 \mathrm{~cm}$ above its base. Chicks will spontaneously peck at the bead and indicate that they have tasted the MeA by showing one or more components of the disgust response, i.e., head shaking, beak wiping, and eye closure. Control chicks were presented with a red bead dipped in distilled water. The latency to the first peck was recorded, and those chicks that failed to peck the bead in $60 \mathrm{sec}$ were excluded from further investigation.

Three hours after training, a retention test was performed. Each chick was presented with a red bead, followed $5 \mathrm{~min}$ later by a similar sized blue bead, both coated with distilled water. The latency to peck each bcad was recorded. If a chick failed to peck either bead within $60 \mathrm{sec}$ of its presentation, the test was terminated. Unpublished experiments in our laboratory have revealed that the color of the training bead (red or blue) has no effect on the chick's subsequent performance and that there is no effect of varying the order of presentation of the red and blue bead at test. Therefore, for simplicity, the chicks were trained on a single color of bead (red), and this was presented first in the test. Both training and testing were performed under a fume hood to minimize possible olfactory effects of $\mathrm{MeA}$. The chicks' behavior was viewed in a mirror suspended above the arena.

Histology. After testing, all experimental chicks were decapitated and their brains were fixed by immersion in $10 \%$ formol saline for at least $60 \mathrm{hr}$. Frozen $40 \mu \mathrm{m}$ serial sections were cut and stained with cresyl violet. The lesions were reconstructed from the histological material by projecting the images of sections through a photographic enlarger onto drawings from an unpublished atlas. The volume of each lesion was estimated from the drawings using a digitizing tablet connected to a microcomputer. The histological sections were examined blind, and those brains that did not contain bilateral lesions in the correct location were discarded.

Statistical analysis. The gender of each chick was determined by the feather-sexing technique (see Hann, 1966). The numerical code was broken, and the results were compared using the $x^{2}$ test with Yates' (1934) correction.

\section{Results}

Significantly more sham-operated chicks trained on a MeAcoated bead avoided an identical water-coated bead at test than did sham-operated chicks trained on a water-coated bead (Fig. $1 ; \chi^{2}=18.95, p<0.001$ ). Similarly, significantly more LCAlesioned chicks trained on MeA avoided the test bead than did LCA-lesioned chicks trained on water (Fig. $1 ; \chi^{2}=6.77, p<$ 0.01 ). There was no significant difference in avoidance of the red bead at test between sham-operated and LCA-lesioned chicks trained on either MeA or water (Fig. 1). Thus, both shamoperated and LCA-lesioned chicks trained on MeA, learned the

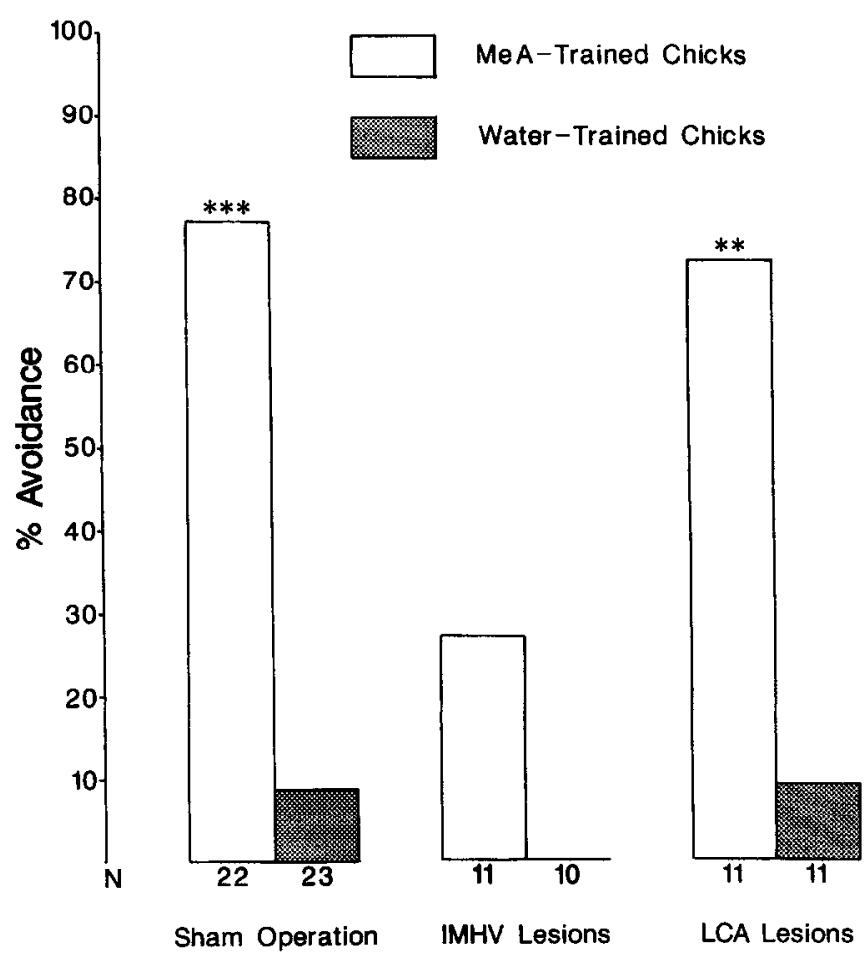

Figure 1. Comparison of the effects of bilateral IMHV lesions, LCA lesions, and sham operation on 1-trial PAL. The chicks were trained 15-20 hr after surgery and were given a retention test $3 \mathrm{hr}$ later. All IMHV-lesioned chicks trained on the water-coated bead pecked the red bead in the test ( $0 \%$ avoidance). $N$ is given beneath each bar; ${ }^{* *} p<$ $0.01,{ }^{* * *} p<0.001$.

1-trial PAL task. There was no difference between these treatments.

In contrast, there was no significant difference in avoidance of the red bead at test between IMHV-lesioned chicks trained on MeA or on water (Fig. 1). Neither was there any difference in avoidance of the red bead at test between IMHV-lesioned, LCA-lesioned, or sham-operated chicks trained on water. However, IMHV-lesioned, MeA-trained chicks avoided the red bead at test significantly less than did similarly trained LCA-lesioned and sham-operated chicks $\left(\chi^{2}=6.42, p<0.02\right.$ for LCA-lesioned and sham-operated groups combined). Thus, IMHV-lesioned chicks did not learn the 1-trial PAL task.

Failure to peck the bead during training (and therefore subsequent exclusion from further investigation) was not affected by the chicks' treatment or training substance. In those chicks that pecked the bead during training, the latency to peck either the MeA- or water-coated bead did not differ between shamoperated and lesioned chicks. Thus, during training all groups of chicks showed similar pecking activity. There was no significant difference in the pecking of male and female chicks in any of the groups studied.

\section{Bead color discrimination}

Sham-operated, MeA-trained chicks avoided the red bead significantly more than the blue bead at test (Table $1 ; \chi^{2}=4.61$, $p<0.05$ ). Similarly, there was a significantly greater avoidance of the red bead then the blue bead at test by LCA-lesioned chicks trained on MeA (Table $1 ; \chi^{2}=6.77, p<0.01$ ). Therefore, those sham-operated and LCA-lesioned chicks trained on MeA that learned the 1-trial PAL task, could discriminate between the 

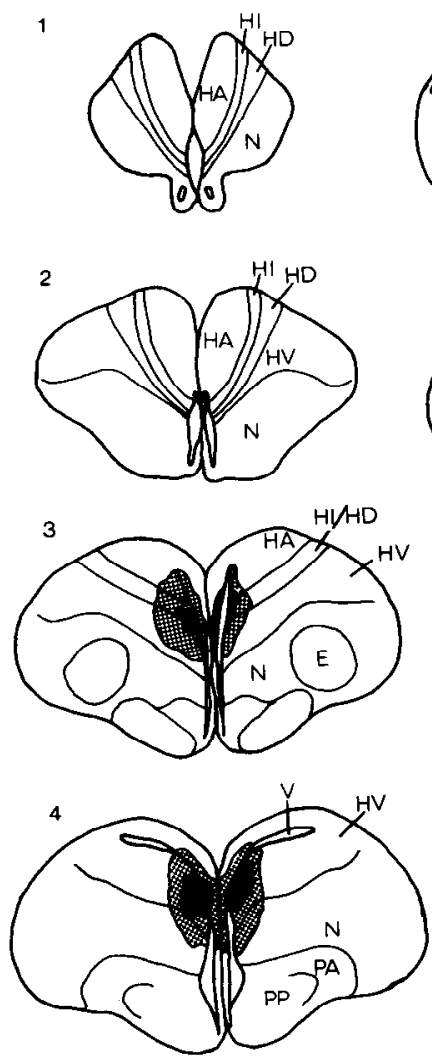

IMHV Lesions
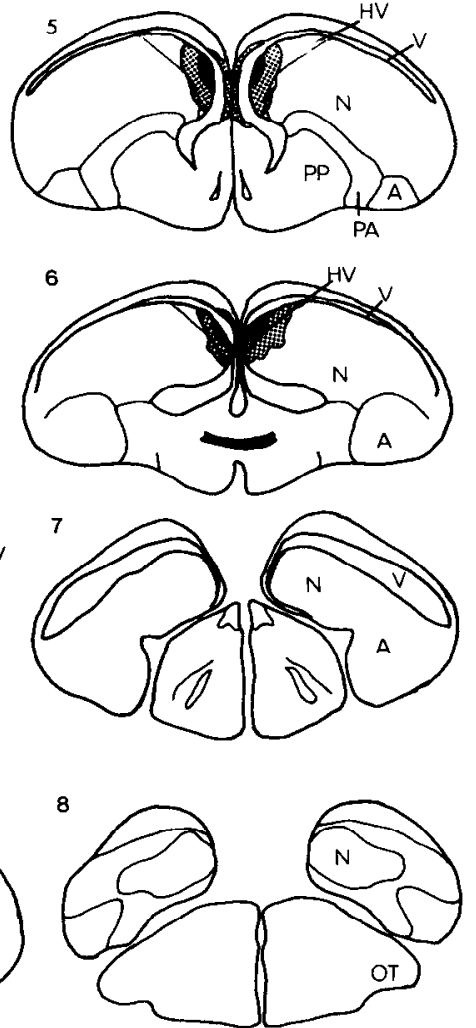

$10 \mathrm{~mm}$
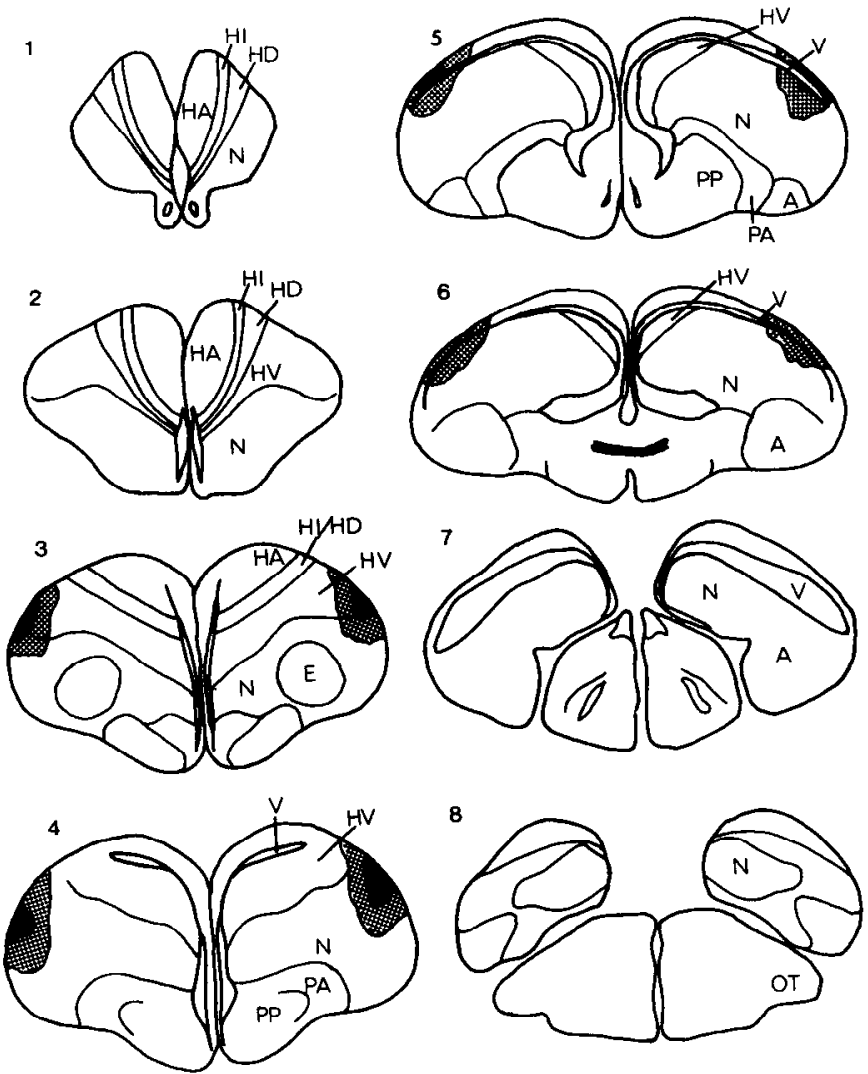

LCA Lesions

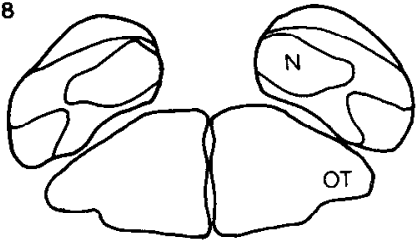

$10 \mathrm{~mm}$

Figure 2. Histological reconstruction of forebrain lesions on diagrams of coronal brain sections. The diagrams represent sections approximately $1 \mathrm{~mm}$ apart. Black areas indicate tissue destroyed in $90 \%$ of brains, and stippled areas denote tissue destroyed in more than $25 \%$ of brains. Section 1 is anterior and section 8 posterior. The left hemisphere is shown on the left side. Abbreviations: $H A$, hyperstriatum accessorium; $H I$, hyperstriatum intercalatus; $H D$, hyperstriatum dorsale; $N$, neostriatum; $H V$, hyperstriatum ventrale; $E$, ectostriatum; $V$, ventricle; $P A$, paleostriatum augmentatum; $P P$, paleostriatum primitivum; $A$, archistriatum; $O T$, optic tectum.

bead on which they were trained and a similar-sized bead of a different color. However, there was no significant difference between the avoidance of the red and blue bead at test by IMHVlesioned chicks trained on MeA. These chicks showed low avoidance of both beads (Table 1). There was no significant difference in avoidance of the blue bead at test, between the 3 experimental groups trained on a MeA-coated bead. Sham-operated, LCA-lesioned, and IMHV-lesioned chicks trained on water all showed a similar low avoidance of both the red and blue beads at test (Table 1).

Table 1. Comparison of the effects of bilateral IMHV lesions, LCA lesions, and sham operation on bead color discrimination at test

\begin{tabular}{|c|c|c|c|c|}
\hline \multirow{2}{*}{$\begin{array}{l}\text { Training } \\
\text { substance }\end{array}$} & \multirow[b]{2}{*}{ Lesion site } & \multirow[b]{2}{*}{$N$} & \multicolumn{2}{|c|}{$\%$ Avoidance at test } \\
\hline & & & Red bead & Blue bead \\
\hline MeA & IMHV & 11 & 27.3 & 9.1 \\
\hline MeA & LCA & 11 & $72.7^{* *}$ & 9.1 \\
\hline $\mathrm{MeA}$ & Sham & 22 & $77.3^{*}$ & 40.9 \\
\hline Water & IMHV & 10 & 0 & 0 \\
\hline Water & LCA & 11 & 9.1 & 27.3 \\
\hline Water & Sham & 23 & 8.7 & 8.7 \\
\hline
\end{tabular}

The percentage of chicks avoiding the red and blue water-coated beads at test is given. All chicks were trained on a red bead. For each group, the percentage of chicks avoiding the red bead and the percentage of chicks avoiding the blue 'vead in the test is compared; ${ }^{*} p<0.05,{ }^{* *} p<0.01$.

\section{Anatomical location of lesions}

Reconstructions of the IMHV and LCA lesions are shown in Figure 2. The volume of tissue destroyed, expressed as a percentage of total forebrain volume ( \pm SEM), was $3.06 \pm 0.38 \%$ $(N=21)$ for IMHV and $2.19 \pm 0.13 \%(n=22)$ for LCA. IMHVlesioned, MeA-trained chicks that did not learn the 1-trial PAL task received lesions of similar size and location to those in chicks that did learn the task. For both lesion groups, the extent of the damage was the same in each hemisphere, and there was no significant difference (Mann-Whitney $U$ test) in the amount of tissue destroyed.

\section{Discussion}

Bilateral lesions placed in IMHV before training impaired 1-trial PAL. In contrast, sham-operated and bilateral LCA-lesioned chicks were able to learn the task. It is unlikely that the learning impairment resulting from IMHV lesions can be attributed to general effects of anesthesia or nonspecific brain damage since (1) sham-operated and LCA-lesioned chicks were not impaired in the task; (2) there was no difference in the latency to peck during training between IMHV-lesioned chicks and the other groups. Furthermore, all lesioned groups of chicks showed low avoidance of the blue bead at test.

Lesion studies have previously been employed in the investigation of 1-trial PAL in chicks, but to our knowledge the current study is the first to use such restricted forebrain lesions. 
In an early attempt to discern which forebrain regions are involved in 1-trial PAL, Benowitz (1972) selected relatively gross morphological regions for suction lesions. Both "limited" and "extensive" hyperstriatal lesions were found to impair acquisition of the task, while "extensive" lesions alone impaired retention. The "hyperstriatum accessorium, -dorsalis, -intercalatus and overlying paleocortical tissue" were removed by both operations, and the "extensive" lesions "more frequently included hyperstriatum ventrale, parts of adjacent neostriatum and medial portions of the hippocampal complex." Thus, from the study of Benowitz (1972), it was impossible to associate any particular forebrain structure with the learning process. The results of the present study indicate that a specific part of the hyperstriatum ventrale, the IMHV, is critically involved in the acquisition of a 1-trial PAL task. Patterson et al. (1986) recently demonstrated that bilateral injection of a variety of agents (L-glutamate, ouabain, cyclohexamide, anisomycin, emetine) into the MHV prevents chicks from learning a 1-trial PAL task. Our findings are in agreement with these results but specify more precisely the region critically involved.

Bilateral IMHV lesions have previously been shown to severely impair the ability of chicks to acquire (McCabe et al., 1981) and retain (McCabe et al., 1982) an imprinted preference. Thus, IMHV appcars to play a critical role in both filial imprinting and in the acquisition of a 1-trial PAL task in the young chick. Furthermore, the part of the MHV implicated in 1-trial PAL by Rose and coworkers (see Rose, 1985) and Patterson et al. (1986) appears to be, or at least to include, IMHV.

Bilateral LCA lesions did not affect the acquisition of a 1-trial PAL task in the present study. These lesions damaged the lateral edges of the hyperstriatum ventrale and the dorsolateral part of the neostriatum and its overlying cortical mantle. Lesions similar in location and extent to these have also been shown not to affect the retention of an imprinted preference (McCabe et al., 1982; Horn et al., 1983). Removal of the lateral neostriatum has also been shown to have no effect on imprinting in ducklings (Takamatsu and Tsukada, 1985). These findings are perhaps not surprising since biochemical experiments have not implicated this area in either imprinting (Horn et al., 1979) or 1-trial PAL (Kossut and Rose, 1984).

However, more extensive suction lesions of the chick lateral forebrain have been reported to affect both the acquisition and retention of an imprinted preference (Salzen et al., 1975, 1978) and the acquisition and retention of a 1-trial PAL task (Benowitz, 1972). In both of these studies the lesions included at least part of the archistriatum. The avian archistriatum is believed to have a role in motivation and emotion (Zeier and Karten, 1971) and has reciprocal connections with IMHV (Bradley et al., 1985). Thus, it is possible that damage to this structure may cause "motivational" deficits that could affect the chicks' ability to learn. In fact, Benowitz (1980) cites his own unpublished observations that destruction of the chicks' medial archistriatum causes PAL deficits similar to those resulting from lateral forebrain lesions.

Patterson et al. (1986) found that direct injection of amnestic drugs into the lateral neostriatum (LNS) prevented chicks from learning a 1-trial PAL task. From the description given, the injection site would seem to be below LCA, but its precise relationship to the archistriatum is not clear.

Experiments employing unilateral injections of amnestic agents (Patterson et al., 1986) have led to the suggestion that it is the left MHV/IMHV and right LNS that are the critical structures involved in the learning of a 1-trial PAL task. Hemispheric asymmetries in the neural correlates of imprinting (Bradley et al., 1981; Horn et al., 1985) and 1-trial PAL (Stewart et al., 1984) are well established, and Horn et al. (1983) have suggested that there is a temporal processing of information during imprinting between different brain regions. It is therefore possible that there is a time-ordered movement of information during 1-trial PAL between left IMHV and right LNS or, as Patterson et al. (1986) suggest, that processing of the emotional aspects of 1-trial PAL occurs in the right LNS and associative processing occurs in the left IMHV. These possibilities remain to be tested:

In the 1-trial PAL task employed in the current experiments, chicks may associate pecking a bead with an unpleasant taste and subsequently avoid the bead. Bilateral lesions to IMHV prevented the acquisition of this 1-trial PAL task. This finding appears to conflict with the results of previous studies in which bilateral IMHV lesions did not impair the acquisition of other associative learning tasks but nevertheless did prevent imprinting (McCabe et al., 1982; Johnson and Horn, 1986). However, there are 2 characteristics of this 1-trial PAL task that may account for the effects of the IMHV lesions. First, the task requires the chick to inhibit its response to a stimulus, and second, the task may involve recognition of the characteristics of an object. The learning process of imprinting may involve both response inhibition and object recognition, but the learning tasks in which IMHV lesions are not amnestic do not.

When a chick is presented with a small conspicuous bead, it will normally try to peck at it. This tendency is inhibited as a result of exposure to a bead coated in a noxious substance such as MeA. Imprinting may be characterized in a similar way. When a naive chick is exposed to an appropriately sized visually conspicuous object, it will approach the object. The longer the time that the chick is exposed to this initial object, the less it will subsequently approach different objects. Thus, imprinting may involve the selective inhibition of a tendency to approach. Following this interpretation, IMHV may be involved in the inhibition of an inborn tendency in both 1-trial taste aversion PAL and imprinting. In neither heat-reinforced pattern discrimination (McCabe et al., 1982), nor the acquisition of an operant pcdal-pressing task (Johnson and Horn, 1986), is response inhibition required, and bilateral IMHV lesions do not affect the acquisition of these tasks. However, reversals of these tasks may be sensitive to IMHV lesions.

In the present study a significant number of sham-operated and LCA-lesioned chicks trained on a red MeA-coated bead avoided the red bead but pecked at a blue bead in the test. If the chicks had simply associated the act of pecking with the aversive taste and not retained any information about the characteristics of the training bead, they would have avoided both the red and blue beads in the test. Thus, the chicks appear to store information about the characteristics of the training bead. This conclusion is supported by a recent study in which the strength of initial learning and duration of the memory of a 1 -trial PAL task in chicks were found to be dependent upon the visual characteristics of the training bead (Roper and Redston, 1987).

Johnson and Horn (1986) have proposed that there are at least 2 types of dissociable memory system in chicks, one concerned with the recognition of objects and the other with the acquisition of simple associations. Furthermore, they have argued that the integrity of IMHV is essential for the former system but not for the latter. If this is the case, then bilateral 
lesions to IMHV should impair the chicks' ability to recognize a particular bead. In the present experiments, the inability to recognize the red training bead may prevent the association between the bead and the unpleasant taste from being recalled in the test, resulting in the amnesia observed in IMHV-lesioncd chicks.

\section{References}

Bateson, P. P. G. (1966) The characteristics and context of imprinting. Biol. Rev. 41: 177-220.

Benowitz, L. (1972) Effects of forebrain ablations on avoidance learning in chicks. Physiol. Behav. 9: 601-608.

Benowitz, L. (1980) Functional organisation of the avian telencephalon. In Comparative Anatomy of the Telencephalon, S. O. E. Ebbesson, ed., pp. 389-421, Plenum, New York.

Bradley, P., D. C. Davies, and G. Horn (1985) Connections of the hyperstriatum ventrale in the domestic chick (Gallus domesticus). J. Anat. 140:577-589.

Bradley, P., G. Horn, and P. Bateson (1981) Imprinting: An electron microscopic study of chick hyperstriatum ventrale. Exp. Brain Res. 41: $115-120$

Burgoyne, R. D., and S. P. R. Rose (1980) Subcellular localisation of increased incorporation of $[3 \mathrm{H}]$ fucose following passive avoidance learning in the chick. Neurosci. Lett. 19: 343-348.

Cherkin, A. (1969) Kinetics of memory consolidation. Role of amnesic treatment parameters. Proc. Natl. Acad. Sci. USA 63: 1094-1101.

Davies, D. C., and G. Horn (1983) Putative cholinergic afferents of the chick hyperstriatum ventrale: A combined acetylcholinesterase and retrograde fluorescence labelling study. Neurosci. Lett. 38: 103107.

Hann, C. M. (1966) Sex Linkage in Poultry Breeding. Min. Agric. Food Fish. Bull. 38: 6-7.

Horn, G. (1981) Neural mechanisms of learning: An analysis of imprinting in the domestic chick. Proc. R. Soc. London [Biol.] 213:101137.

Horn, G. (1985) Memory, Imprinting and the Brain, Clarendon, Oxford, UK.

Horn, G., B. J. McCabe, and P. P. G. Bateson (1979) An autoradiographic study of the chick brain after imprinting. Brain Res. 168:361373.

Horn, G., B. J. McCabe, and J. Cipolla-Neto (1983) Imprinting in the domestic chick: The role of each side of the hyperstriatum ventrale in acquisition and retention. Exp. Brain Res. 53: 91-98.

Horn, G., P. Bradley, and B. J. McCabe (1985) Changes in the structure of synapses associated with learning. J. Neurosci. 5: 3161-3168.

Johnson, M. H., and G. Horn (1986) Dissociation of recognition memory and associative learning by a restricted lesion of the chick forebrain. Neuropsychologia 24: 329-340.

Johnson, M. H., and G. Horn (1987) The role of a restricted region of the chick forebrain in the recognition of individual conspecifics. Behav. Brain Res. 23: 269-275.

Kohsaka, S., K. Takamatsu, E. Aoki, and Y. Tsukada (1979) Metabolic mapping of chick brain after imprinting using [14C] 2-deoxyglucose technique. Brain Res. 172: 539-544.

Kossut, M., and S. P. R. Rose (1984) Differential 2-deoxyglucose uptake into chick brain structures during passive avoidance training. Neuroscience 12: 971-977.

Mason, R. J., and S. P. R. Rose (1987) Lasting changes in spontaneous multi-unit activity in the chick brain following passive avoidance learning. Neuroscience 21: 931-942.

McCabe, B. J., G. Horn, and P. P. G. Bateson (1981) Effects of restricted lesions of the chick forebrain on the acquisition of filial preferences during imprinting. Brain Res. 205: 29-37.

McCabe, B. J., J. Cipolla-Neto, G. Horn, and P. Bateson (1982) Amnesic effects of bilateral lesions placed in the hyperstriatum ventrale of the chick after imprinting. Exp. Brain Res. 48: 13-21.

Patterson, T. A., M. C. Alvarado, I. T. Warner, E. L. Bennet, and M. R. Rosenzweig (1986) Memory stages and brain asymmetry in chick learning. Behav. Neurosci. 100: 856-865.

Payne, J. K., and G. Horn (1982) Differential effects of exposure to an imprinting stimulus on "spontaneous" neuronal activity in two regions of the chick brain. Brain Res. 232; 191-193.

Payne, J. K., and G. Horn (1984) Long-term consequences of exposure to an imprinting stimulus on "spontaneous" impulse activity in the chick brain. Behav. Brain Res. 13: 163-172.

Roper, T. J., and S. Redston (1987) Conspicuousness of distasteful prey affects the durability of one-trial avoidance learning. Anim. Behav. 35: 739-747.

Rose, S. P. R. (1985) The cell biological consequences of passive avoidance training in the chick. In Brain Plasticity, Learning and Memory, B. E. Will, P. Schmitt, and J. C. Dalrymple-Alford, eds., pp. 39-50, Plenum, New York.

Rose, S. P. R. (1987) Long-term memory formation is blocked by 2-deoxygalactose, a fucose analogue. Neurosci. Lett. Suppl. 29: 102.

Salzen, E. A., D. M. Parker, and A. J. Williamson (1975) A forebrain lesion preventing imprinting in domestic chicks. Exp. Brain Res. 24: 145-157.

Salzen, E. A., D. M. Parker, and A. J. Williamson (1978) Forebrain lesions and retention of imprinting in domestic chicks. Exp. Brain Res. 31: 107-116.

Stewart, M. G., S. P. R. Rose, T. S. King, P. L. A. Gabbott, and R. Bourne (1984) Hemispheric asymmetry of synapses in chick medial hyperstriatum ventrale following passive avoidance training: A stereological investigation. Dev. Brain. Res. 12: 261-269.

Sukumar, R., S. P. R. Rose, and R. D. Burgoyne (1980) Increased incorporation of $[3 \mathrm{H}]$ fucose into chick brain glycoproteins following training on a passive avoidance task. J. Neurochem. 34: 1000-1006.

Takamatsu, K., and Y. Tsukada (1985) Neurochemical studies on imprinting behavior in chick and duckling. Neurochem. Res. 10: 1371-1391.

Yates, F. (1934) Contingency tables involving small numbers and the $\chi^{2}$ test. J. R. Statist. Soc. Suppl. 1: 217-235.

Zeier, H., and H. J. Karten (1971) The archistriatum of the the pigeon: Organisation of afferent and efferent connections. Brain Res. 31:313326. 